\title{
Ensino da Inteligência Artificial na Educação Básica: um novo horizonte para as pesquisas brasileiras
}

\author{
Marcos Yuzuru de Oliveira Camada e Gilvan Martins Durães
}

Grupo de Pesquisa em Sistemas e Computação (GPESC) - Campus Catu - Instituto Federal de Educação, Ciência e Tecnologia Baiano - (IF BAIANO)

Caixa Postal 15.064 - 91.501-970 - Catu - BA - Brazil

\{marcos.camada, gilvan.duraes\} @ifbaiano.edu.br

\begin{abstract}
Some works have been proposed over the last few years to presents the teaching of Artificial Intelligence (AI) in basic education. This paper discusses the results of a systematic research that uses Brazilian and international databases in order to shown the state of the art in the field and verify the relevance of that approach for the Brazilian Basic Education by assumptions of the National Curricular Common Base. The results point to possibilities of works and application of teaching AI in Brazilian Basic Education.
\end{abstract}

Resumo. Nos últimos anos alguns trabalhos propuseram o ensino de fundamentos da Inteligência Artificial (IA) na Educação Básica. Este artigo apresenta e discute os resultados de uma pesquisa sistemática utilizando bases de dados cientificas nacionais e internacionais com o objetivo de apresentar o estado da arte na área e verificar a relevância da abordagem para a Educação Básica no Brasil a partir dos pressupostos da Base Nacional Comum Curricular (BNCC). Os resultados apontam para as possibilidades de pesquisa e aplicação do ensino da IA na Educação Básica Brasileira.

\section{Introdução}

Nos últimos anos o ensino da matemática básica no Brasil tem passado por uma transformação através da melhor compreensão e maior uso das tecnologias digitais da informação e comunicação na educação, favorecendo o interesse e o desenvolvimento das chamadas competências do Século XXI, tais como utilização de informação, criatividade, pensamento sistêmico, investigação e pesquisa [Inep 2019, OCDE 2020]. Dentre as transformações curriculares encontra-se o desenvolvimento do Pensamento Computacional previsto no currículo da Base Nacional Comum Curricular (BNCC) [Brasil 2018].

O termo Pensamento Computacional (PC) vem do Inglês Computational Thinking, o qual se popularizou com a publicação, em 2006, do artigo "Computational Thinking" da pesquisadora Jeanette M. Wing. A autora defende a ideia de que o PC é uma habilidade essencial para todos, assim como ler, escrever e calcular [Wing, 2006]. Em 2011, a International Society for Technology in Education (ISTE) juntamente com a Computer Science Teachers Association (CSTA) trabalharam com professores de Ciência da Computação e das áreas de Humanas para definir um conceito para o PC com a finalidade de nortear as atividades realizadas na Educação Básica [Valente 2016].

O grupo ISTE/CSTA [ISTE, 2020] definiu então que o Pensamento Computacional é um processo de resolução de problemas que inclui (mas não está limitado a) as seguintes características: formulação de problemas de forma que nos permita usar um computador e outras ferramentas para nos ajudar a resolvê-los; organização e análise lógica dos dados; representação de dados através de abstrações, como modelos e simulações; automatização de soluções através do pensamento algorítmico; identificação, análise e implementação de 
possíveis soluções com o objetivo de alcançar a combinação mais eficiente e efetiva de etapas e recursos; generalização e transferência deste processo de resolução de problemas para uma grande variedade de problemas. A partir desta definição geral do PC, é possível expandi-la para as características básicas da Inteligência Artificial (IA) como sendo: autonomia e adaptabilidade [Russel e Norvig 2020].

A motivação deste trabalho parte da mobilização internacional e, em menor proporção, nacional, para a inclusão, não apenas do PC, mas também dos fundamentos da IA na Educação Básica [SBC 2019]. Neste sentido, aliado ao crescimento da importância da IA na economia global e nas oportunidades do mundo do trabalho, a Organização para a Cooperação e Desenvolvimento Econômico (OCDE) tem incluído o PC juntamente com o pensamento criativo como competência requerida para a Educação Básica em seus componentes de estudos e proposta para o PISA 2021 [OCDE 2020].

No contexto do PC e do pensamento criativo, a IA pode ser compreendida como uma área de estudo e aplicação computacional do raciocínio lógico para a resolução de problemas e/ou predição em meio à incerteza [Russel e Norvig 2020]. Por ser uma área cada vez mais abrangente no dia a dia da sociedade, seja na automação de atividades em casa, num e-mail classificado como spam, num atendimento virtual via chatbot, numa recomendação recebida ao navegar pela internet, e até mesmo na organização da educação, a compreensão dos fundamentos da IA desde a Educação Básica tende a ter um amplo interesse [Gomes e Balmant 2019, Mcelwee 2019, Cimm 2020].

No Brasil, as principais entidades mobilizadoras do ensino e utilização do Pensamento Computacional são a Sociedade Brasileira de Computação (SBC) e o Centro de Inovação para a Educação Brasileira (CIEB). Estas entidades estão se mobilizando para inclusão efetiva de competências do Pensamento Computacional na BNCC [SBC 2019ab, CIEB 2020]. Por outro lado, as pesquisas relacionadas ao ensino dos fundamentos da Inteligência Artificial na Educação Básica brasileira ainda são preliminares.

Este artigo apresenta uma visão geral da potencialidade do ensino da IA na Educação Básica brasileira a partir de pressupostos da BNCC, através do estímulo ao Pensamento Computacional. Além disso, este artigo apresenta e discute os resultados de uma revisão sistemática, com o objetivo de fomentar as pesquisas e as aplicações do ensino de fundamentos da IA na Educação Básica brasileira.

Este artigo está organizado da seguinte forma. Na Seção 2, são apresentados os trabalhos relacionados; na Seção 3, são apresentados os fundamentos e motivações do ensino da IA na Educação Básica brasileira; a Seção 4, apresenta e discute os resultados da Revisão Sistemática realizada; por fim, a Seção 5 apresenta as considerações finais da área.

\section{Trabalhos relacionados}

Os seguintes trabalhos relacionados foram elencados [Queiroz, Sampaio e Lima, 2017; Touretzky et al., 2019; Loftus e Madden, 2020].

Loftus e Madden (2020) apresentam uma abordagem construtivista hands-on para o ensino de fundamentos da Aprendizagem de Máquina e da Inteligência Artificial, utilizando uma aplicação das Redes Bayesianas onde os estudantes são envolvidos no processo de construção e discussão dos dados. O projeto de ensino foi aplicado em uma turma de estudantes do primeiro ano do módulo IoT de um bacharelado em computação. Apesar deste trabalho ter o foco no ensino superior, a abordagem dos autores pode ser estendidas e adaptada para a Educação Básica, pois provê, a partir de um conceito fundamental da IA [Darwiche 2010], a 
"leitura de mundo" [Freire 1970], a qual também é essencial na Educação Básica e está implicitamente prevista na BNCC.

Queiroz et al. (2019) realizam uma investigação sobre estratégias a serem adotadas para o aprendizado de Inteligência Artificial no Ensino Fundamental por meio da robótica educacional. A pesquisa de revisão bibliográfica apresentada em [Queiroz et al., 2017] identificou apenas um trabalho diretamente associado ao ensino da IA na Educação Básica, o qual defende a "alfabetização em IA", a partir dos primeiros anos escolares e propõe uma base curricular em IA para cada fase escolar (Infantil, Fundamental I e II, e Ensino Médio).

Em [Touretzky et al. 2019], são apresentados os avanços no último ano no interesse e na produção acadêmica na área de Ensino da IA na Educação Básica (EB) e apresenta o Projeto AI4K12 que tem como objetivo definir diretrizes nacionais para o Ensino da IA na EB dos EUA e reunir uma comunidade aberta de desenvolvedores de recursos e ferramentas para a IA na EB. O AI4K12 é um projeto da Association for the Advancement of Artificial Intelligence (AAAI) e financiado pela National Science Foundation (AI4K12.org).

\section{Ensino do PC na Educação Básica Brasileira e Pontes para o Ensino da IA}

Segundo a BBC (2018), o PC está organizado em quatro pilares interdependente. Cada pilar é essencial para o outro, assim como as "pernas" de uma mesa, para dar sustentabilidade durante todo o processo de resolução do problema. A literatura distingue os 4 (quatro) pilares do PC [Wing 2006]: Decomposição, Reconhecimento de Padrões, Abstração e Algoritmos.

O ensino do PC na Educação Básica (EB) vem se popularizando no Brasil principalmente a partir de portais públicos de educação [CIEB 2020, SBC, 2019, Brackmann 2020]. Recentemente a SBC investiu esforços em produzir documentos norteadores para o ensino da computação no Brasil. Tais documentos estão alinhados com a BNCC e tem como principal objetivo delinear o currículo de formação em computação mobilizando competências em diversas áreas [SBC 2019b]. Destaca-se que estão distribuídos neste currículo proposto pela $\mathrm{SBC}$ todos os quatro pilares radicais do $\mathrm{PC}$.

A BNCC prevê em seu documento, na seção de competências específicas e habilidades da matemática e suas tecnologias, as seguintes habilidades:

\footnotetext{
(EM13MAT405) Utilizar conceitos iniciais de uma linguagem de programação na implementação de algoritmos escritos em linguagem corrente e/ou matemática [Brasil 2018, p. 544].

(EM13MAT315) Investigar e registrar, por meio de um fluxograma, quando possível, um algoritmo que resolve um problema [Brasil 2018, p. 544].
}

Desta forma, o PC aqui representado pelos algoritmos em geral, está previsto como habilidade a ser desenvolvida no currículo da matemática e suas tecnologias. Ademais, dentre as dez competências gerais que orientam a BNCC [Brasil 2018], podemos destacar duas competências que estão relacionadas diretamente ao tema em questão:

2. Exercitar a curiosidade intelectual e recorrer à abordagem própria das ciências, incluindo a investigação, a reflexão, a análise crítica, a imaginação e a criatividade, para investigar causas, elaborar e testar hipóteses, formular e resolver problemas e criar soluções (inclusive tecnológicas) com base nos conhecimentos das diferentes áreas [Brasil 2018, p.9].

6. Valorizar a diversidade de saberes e vivências culturais e apropriar-se de conhecimentos e experiências que the possibilitem entender as relações próprias do mundo do trabalho e fazer escolhas alinhadas ao exercício da cidadania e ao seu projeto de vida, com liberdade, autonomia, consciência crítica e responsabilidade [Brasil 2018, p.9]. 
Observa-se nessas competências a viabilidade do uso do PC como meio para que os estudantes da Educação Básica consigam desenvolver suas habilidades e terem uma formação de atitudes e valores, de acordo a Lei de Diretrizes e Bases da Educação Nacional [Brasil 1996]. Assim, o PC vai além da área da Computação ao permitir desenvolver a criatividade e a autonomia dos discentes na solução de problemas em geral e na melhor compreensão do mundo ao seu redor. Muitos trabalhos de pesquisa estudam e propõem recursos educacionais para o ensino e aprendizagem do PC na Educação Básica [Brackmann 2017 e 2020, Meira 2017, CIEB 2020]. Ainda na seção supracitada da BNCC, consta como habilidades

(EM13CNT205) Interpretar resultados e realizar previsões sobre atividades experimentais, fenômenos naturais e processos tecnológicos, com base nas noções de probabilidade e incerteza, reconhecendo os limites explicativos das ciências [Brasil 2018, p. 557].

Tal habilidade de realizar previsões baseadas em noções de probabilidade e incerteza está diretamente relacionada aos fundamentos da IA que conceitua os sistemas classificadores, sistemas de reconhecimento de padrões e sistemas preditivos, baseados em análise de dados do passado para fazer inferências normalmente do futuro [Olivo, Santin, e Oliveira 2015, Gomes e Balmant 2019, Mcelwee 2019, Russel e Norvig 2020]. Além disso, segundo a BNCC as finalidades do ensino médio incluem

\begin{abstract}
prever o suporte aos jovens para que reconheçam suas potencialidades e vocações, identifiquem perspectivas e possibilidades, construam aspirações e metas de formação e inserção profissional presentes e/ou futuras, e desenvolvam uma postura empreendedora, ética e responsável para transitar no mundo do trabalho e na sociedade em geral [Brasil 2018, p. 466].
\end{abstract}

Assim, é fortemente desejável que os jovens da contemporaneidade compreendam o mundo digital e suas tecnologias (especificamente o Pensamento Computacional e os fundamentos da Inteligência Artificial) os quais os envolve e permeia o mundo do trabalho e a sociedade em geral. Desta forma, os esforços a serem realizados para o ensino da IA contribuirão com a educação integral do ser humano ao propor a superação da fragmentação dos processos pedagógicos na qual disciplinas não interagem entre si e nem relacionam o conteúdo delas com o cotidiano do estudante. Neste sentido,

\footnotetext{
Temas Contemporâneos Transversais têm a condição de explicitar a ligação entre os diferentes componentes curriculares de forma integrada, bem como de fazer sua conexão com situações vivenciadas pelos estudantes em suas realidades, contribuindo para trazer contexto e contemporaneidade aos objetos do conhecimento descritos na BNCC [Brasil 2019, p. 5].
}

Vale ressaltar que o tema Inteligência Artificial (IA) se enquadra na macroárea de temas contemporâneos transversais Ciência e Tecnologia, de acordo com as definições da Secretaria de Educação Básica do Ministério da Educação [Brasil 2018 e 2019].

IA é uma subárea da Computação com muitas definições e subjetividades ao longo da sua história de quase um século. O chamado Teste de Turing proposto em 1950 já apontava para a subjetividade do "o que é pensar?". Turing propôs um jogo no qual uma pessoa faria perguntas por escrito a um sistema computacional e tentaria descobrir se quem estaria respondendo seria um ser humano ou a máquina [Russel e Norvig 2020]. Aplicações modernas apontam para um conceito de IA voltado para duas principais características: autonomia e adaptabilidade. Autonomia é a habilidade de executar tarefas em contextos complexos sem constante intervenção do ser humano e adaptabilidade é a habilidade de melhorar seu desempenho aprendendo com a experiência. [Russel e Norvig 2020].

Dentre as aplicações, serviços e algoritmos que se enquadram nestas características da Inteligência Artificial, é possível citar alguns exemplos: sistema que joga xadrez, algoritmo de 
logística, reconhecimento de voz, texto manuscrito, faces e imagens, veículo autônomo, robô que faz atendimento via chat (chatbot); sistemas de recomendações pessoais e de negócio, detecção de spam de e-mail, predições médicas, tais como, predição de complicações cirúrgicas, detecção de sinais de diabetes a partir de coleta de dados pessoais, aperfeiçoamento de modelos matemáticos para pré-diagnóstico do coronavírus [Olivo, Santin e Oliveira 2015, Velazco 2018, Gomes e Balmant 2019, Mcelwee 2019, Canto e Scartezini 2020, Russel e Norvig 2020]. Nota-se a diversidade de áreas e que muitas vezes as pessoas não percebem que estão lidando com máquinas que utilizam IA. Daí a necessidade de se tratar deste assunto cada vez mais cedo na Educação Básica [Pimentel et al. 2018].

Apesar da IA ser uma área de interesse global inclusive para uso nos ambientes educacionais [Moreira, Mesquita e Peres 2019, Gomes e Balmant 2019], esta pesquisa mostra que há poucos trabalhos científicos brasileiros diretamente relacionados ao ensino da IA na EB. Dentro da concepção do PC como estratégia interdisciplinar para alcançar diferentes competências previstas na BNCC e, assim, colaborar com a educação integral, esta pesquisa corrobora para a integração do tema Fundamentos em Inteligência Artificial à Educação Básica brasileira. Esta abordagem é inovadora ao passo que foram encontrados poucos trabalhos científicos fortemente correlacionados a ele conforme apresentam as Seções 2 e 4.

Fundamenta-se tal abordagem, por exemplo, a partir da intradisciplinaridade e interdisciplinaridade de conteúdo sugeridos da matemática e da informática do ensino médio, a qual vai apontar para as bases do conhecimento aplicado à inteligência artificial. Assim, observa-se nos Projetos Pedagógicos de Cursos (PPC) dos cursos técnicos integrados ao ensino médio, o seguinte recorte do conteúdo programático do componente curricular Matemática, distribuído ao longo de três séries (anos): razão, proporção e porcentagem; estatística básica; probabilidade. Ademais, o componente curricular Informática relacionada nos mesmos PPCs inclui o seguinte conteúdo: planilha eletrônica; Internet e e-mail. Este recorte específico do conteúdo dos dois componentes curriculares pode ser trabalhado em um projeto de ensino e aprendizagem com o objetivo de melhor compreender o funcionamento de um algoritmo de inteligência artificial para classificação de e-mails do tipo SPAM [Santin e Oliveira 2015]. Alguns destes algoritmos são baseados no Teorema de Bayes (que por sua vez pode ser abordado na perspectiva da resolução de problemas [Jezuz, Oliveira e Tortola 2016].

Desta forma, os autores deste artigo propõem um portal web para o suporte ao Ensino, à Pesquisa e à Popularização da IA na EB, afinal há muito o que se aprender com a inteligência humana e o futuro indeterminado da IA, especialmente no Brasil ${ }^{1}$ deve ser enfrentado de olhos abertos sem esquecer da tecnodemocracia [Lévy 1993]. O portal em desenvolvimento está disponível em: $<$ https://sites.google.com/view/eduia/>.

\section{Revisão Sistemática}

Esta seção apresenta a revisão sistemática realizada com o objetivo de identificar as principais características das pesquisas na área do ensino da IA voltada para a educação básica. Para isto, o estudo foi organizado em três etapas, seguindo preceitos consolidados da Revisão Sistemática [Gough e Thomas 2017]. Inicialmente, foram definidos os termos de busca e os parâmetros de inclusão e exclusão de trabalhos e, desta forma, efetuada a coleta de trabalhos. Na segunda etapa, os trabalhos filtrados foram analisados um a um. Por fim, a partir dos dados compilados e quantificados, os resultados são apresentados e discutidos.

\footnotetext{
${ }^{1}$ Ver mais em < https://itsrio.org/wp-content/uploads/2020/03/RelatorioAI.pdf>
} 
IX Congresso Brasileiro de Informática na Educação (CBIE 2020)

Anais do XXXI Simpósio Brasileiro de Informática na Educação (SBIE 2020)

\subsection{Coleta de publicações}

Nesta pesquisa sistemática foi utilizada o Portal de Publicações da Comissão Especial de Informática na Educação da Sociedade Brasileira de Computação (SBC/CEIE) [SBC 2020], o Portal de Periódicos CAPES [Brasil 2020b], o Portal do Congresso Internacional Informática Educativa (TISE) e o Portal IEEE exclusivamente para pesquisas de trabalhos do IEEE International Symposium on Computers in Education (SIIE). O SBC/CEIE foi escolhido por incluir publicações da Revista Brasileira de Informática na Educação e dos anais dos seis eventos da área promovidos pela SBC. O Portal de Periódicos CAPES foi escolhido por abarcar os principais veículos de publicação científica no Brasil, incluindo atualmente 462 bases de dados internacionais [Brasil 2020b]. O Portal TISE por abarcar trabalhos de um importante evento internacional na área com possibilidades de receber trabalhos em português e, por fim, o SIIE é um evento da AAAI e foi escolhido por ser um evento de renome internacional na área e não indexado ao Portal CAPES.

Os parâmetros de busca foram os seguintes. O período foi de 01/01/2015 a 30/06/2020. Os termos e formato de busca foram: ("inteligência artificial" or "IA") and ("educação básica" or "educação" or "ensino" or "interdisciplinaridade) e os respectivos termos em inglês: ("artificial intelligence" or "AI") and ("k-12" or "education" or "teaching" or "teach" or "interdisciplinary"). A busca pelos termos "IA" ou "AI" foi restringida aos títulos dos trabalhos. Foram considerados apenas trabalhos do tipo Artigo e que já não tivessem sido coletados (exclusão de duplicados). Desta forma, 248 publicações foram inicialmente coletadas. Na leitura dos títulos e resumos de todos trabalhos da coleta de dados inicial, os critérios de inclusão para análise do trabalho foram: discute a importância geral do ensino da IA; aborda o ensino da IA na Educação Básica; aborda ensino da IA de forma interdisciplinar; aborda estratégia de ensino da IA que pode ser utilizada ou adaptada para a Educação Básica. Durante a leitura de todo o texto de cada artigo, os critérios de exclusão foram: aborda exclusivamente a utilização ou aplicação da IA no ambiente educacional; aborda o ensino de tópicos avançados da IA sem nenhuma perspectiva de adaptação para a Educação Básica. A Figura 1 resume os dados quantitativos da pesquisa bem como ilustra o acúmulo do número de trabalhos analisados por ano de publicação.
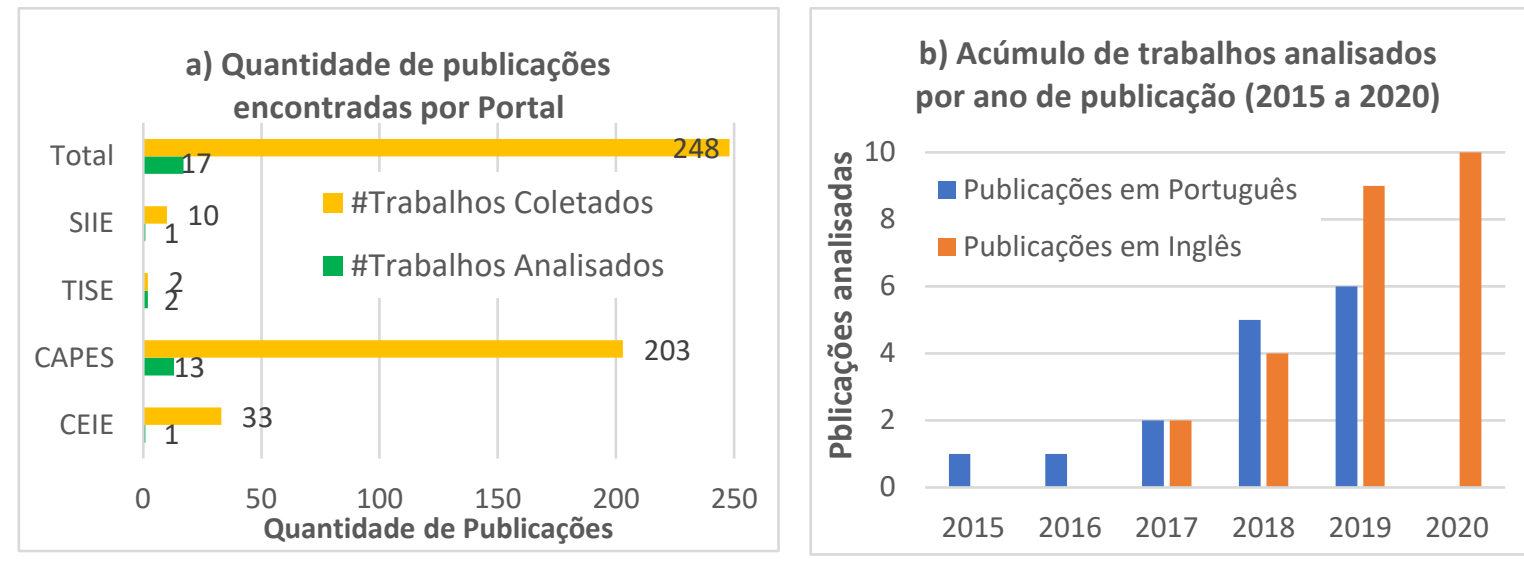

Figura 1. Número de publicações encontradas. Fonte: Próprios Autores.

É possível notar na Figura 1.a que dos 248 trabalhos coletados inicialmente apenas 17 trabalhos (menos de 10\%) foram incluídos na análise da revisão. Vale ressaltar que a maioria dos trabalhos que não foram incluídos para análise trata-se de pesquisas relacionadas ao uso ou aplicação da IA no sistema educacional, a exemplo de propostas de sistemas de tutoria inteligente. Observa-se na Figura 1.b, que dos trabalhos coletados e analisados tinha apenas um publicado até 2016 e que, a partir de 2017, esse número cresceu constantemente. 
IX Congresso Brasileiro de Informática na Educação (CBIE 2020)

Anais do XXXI Simpósio Brasileiro de Informática na Educação (SBIE 2020)

\subsection{Análise das publicações coletadas}

A Tabela 1 apresenta os 17 trabalhos que restaram após aplicação dos critérios de exclusão.

Tabela 1. Lista das publicações analisadas.

\begin{tabular}{|c|c|c|c|}
\hline & Ano & Título & Autor(es) \\
\hline$\# 1$ & 2017 & Inteligência artificial, robôs e ciência aberta & Pretto, Nelson de Luca. \\
\hline \#2 & 2019 & $\begin{array}{l}\text { Resenha crítica do Livro "Trabalho, educação e } \\
\text { inteligência artificial" do Rui Fava }\end{array}$ & Júnior, S. et al. \\
\hline$\# 3$ & 2015 & $\begin{array}{c}\text { Pesquisa e mapeamento de Shells, Frameworks e jogos } \\
\text { para auxiliar no ensino de Inteligência Artificial }\end{array}$ & $\begin{array}{l}\text { Lemos, H. de e Fernandes, } \\
\text { A. M. R. }\end{array}$ \\
\hline$\# 4$ & 2017 & $\begin{array}{c}\text { The Future of Work Is Uncertain, Schools Should } \\
\text { Worry Now; Automation and artificial intelligence are } \\
\text { disrupting the labor market. What do K-12 educators } \\
\text { and policymakers need to know? }\end{array}$ & Herold, Benjamin \\
\hline$\# 5$ & 2017 & $\begin{array}{c}\text { Artificial intelligence, computational thinking, and } \\
\text { mathematics education }\end{array}$ & Gadanidis, $\mathrm{G}$ \\
\hline$\# 6$ & 2018 & $\begin{array}{l}\text { Blue sky ideas in artificial intelligence education from } \\
\text { the EAAI } 2017 \text { new and future AI educator program }\end{array}$ & Eaton, E. et al. \\
\hline$\# 7$ & 2019 & $\begin{array}{c}\text { The solution lies in education: artificial intelligence \& } \\
\text { the skills gap }\end{array}$ & Chrisinger, D. \\
\hline$\# 8$ & 2020 & $\begin{array}{c}\text { The Innovation of Education in the Era of Artificial } \\
\text { Intelligence }\end{array}$ & Shen, J. \\
\hline$\# 9$ & 2019 & Accelerated Move for AI Education in China & Yang, Xiaozhe \\
\hline$\# 10$ & 2017 & $\begin{array}{c}\text { Keeping it Real: Using Real-World Problems to Teach } \\
\text { AI to Diverse Audiences }\end{array}$ & Sintov, N. et al \\
\hline \#11 & 2019 & $\begin{array}{l}\text { BUILT TO TEACH: How URI's AI Lab Blends } \\
\text { Innovation With Learning }\end{array}$ & Kim, B. \\
\hline$\# 12$ & 2018 & Teaching artificial intelligence and humanity & Keating, J., Nourbakhsh, I. \\
\hline$\# 13$ & 2019 & $\begin{array}{c}\text { Using Scratch to Teach Undergraduate Students' Skills } \\
\text { on Artificial Intelligence }\end{array}$ & $\begin{array}{l}\text { Estevez, J., Garate, G., } \\
\text { Guede, J. L., e Graña, M. }\end{array}$ \\
\hline$\# 14$ & 2018 & $\begin{array}{c}\text { Aplicações da Ferramenta de Prototipagem Arduino no } \\
\text { ensino de Inteligência Artificial }\end{array}$ & $\begin{array}{l}\text { Dal Forno, Mateus; Braga, } \\
\text { Paola }\end{array}$ \\
\hline$\# 15$ & 2019 & $\begin{array}{l}\text { Developing Computational Thinking at School with } \\
\text { Machine Learning: An exploration }\end{array}$ & $\begin{array}{l}\text { Rodríguez-García, J. D.; } \\
\text { Moreno-León, J.; Román- } \\
\text { González, M.; Robles, G. }\end{array}$ \\
\hline$\# 16$ & 2018 & $\begin{array}{l}\text { Projeto Frankie: uma proposta para o ensino de } \\
\text { Inteligência Artificial na Educação Básica }\end{array}$ & Pimentel, C. S. et al. \\
\hline$\# 17$ & 2018 & $\begin{array}{l}\text { Ferramentas Computacionais de Ensino de Inteligência } \\
\text { Artificial como Forma de Mitigar Lacunas entre Perfil } \\
\text { de Estudante e de Profissional de TI }\end{array}$ & $\begin{array}{l}\text { Espírito Santo, L. C. E.; } \\
\text { Brancher, J. D. }\end{array}$ \\
\hline
\end{tabular}

Com o objetivo de mapear os trabalhos analisados, atribuiu-se um valor binário (1observado, 0-não observado) para cada uma das seguintes características no artigo: a) Artigo de posicionamento (ensaio); b) Artigo de revisão; c) Artigo de pesquisa aplicada; d) Apresenta portal para difusão científica; e) Propõe repositório de recursos educacionais da IA; f) Apresenta foco explícito na EB; g) Está escrito em português; h) Veículo de publicação apresenta revisão por pares. A Tabela 2 apresenta uma síntese das principais características dos trabalhos com base nesta valoração, na qual a primeira coluna identifica as características listadas anteriormente e a primeira linha identifica o número do trabalho analisado (conforme a Tabela 1). O trabalho de número 18 corresponde a este artigo. Ainda na Tabela 2, a última linha corresponde à quantidade de características observadas em cada artigo, sendo os 
trabalhos de números 15 e 18 os trabalhos que apresentaram maior quantidade de características observadas (6 características presentes).

Destaca-se na Tabela 2 que, dos trabalhos coletados e analisados, apenas 4 trabalhos $(10,13,15$ e 16) apresentam, simultaneamente, as características de ser um artigo de pesquisa primária (pesquisa aplicada) e com foco claro na Educação Básica (características $c$ e f). Destes, apenas o trabalho número 16 é um trabalho de autores brasileiros.

Tabela 2. Mapeamento das publicações analisadas.

\begin{tabular}{|c|c|c|c|c|c|c|c|c|c|c|c|c|c|c|c|c|c|c|}
\hline \# & 1 & 2 & 3 & 4 & 5 & 6 & 7 & 8 & 9 & 10 & 11 & 12 & 13 & 14 & 15 & 16 & 17 & 18 \\
\hline a) & 1 & 1 & 0 & 1 & 1 & 1 & 1 & 1 & 1 & $\mathbf{0}$ & 1 & 1 & 0 & 0 & 0 & 0 & 1 & 1 \\
\hline b) & 0 & 0 & 0 & 0 & 0 & 0 & 0 & 0 & 1 & $\mathbf{0}$ & 0 & 0 & 0 & 0 & 1 & 0 & 1 & 1 \\
\hline c) & 0 & 0 & 1 & 0 & 0 & 0 & 0 & 0 & 0 & 1 & 0 & 1 & 1 & 1 & 1 & 1 & 0 & 0 \\
\hline d) & 0 & 0 & 1 & 0 & 0 & 0 & 0 & 0 & 0 & $\mathbf{0}$ & 0 & 0 & 0 & 0 & 1 & 0 & 0 & 1 \\
\hline e) & 0 & 0 & 0 & 0 & 0 & 0 & 0 & 0 & 0 & $\mathbf{0}$ & 1 & 0 & 0 & 0 & 1 & 0 & 0 & 0 \\
\hline f) & 0 & 1 & 0 & 1 & 1 & 1 & 0 & 1 & 1 & 1 & 0 & 0 & 1 & 0 & 1 & 1 & 0 & 1 \\
\hline g) & 1 & 1 & 1 & 0 & 0 & 0 & 0 & 0 & 0 & 0 & 0 & 0 & $\mathbf{0}$ & 1 & 0 & 1 & 1 & 1 \\
\hline h) & 1 & 1 & 1 & 0 & 1 & 1 & 1 & 1 & 1 & 1 & 0 & 1 & 1 & 1 & 1 & 1 & 1 & 1 \\
\hline Total & 3 & 4 & 4 & 2 & 3 & 3 & 2 & 3 & 4 & 3 & 2 & 3 & 3 & 3 & 6 & 4 & 4 & 6 \\
\hline
\end{tabular}

\subsection{Discussão dos resultados}

Os resultados encontrados na coleta e análise de publicações da revisão sistemática evidenciam o crescente interesse na área de ensino da Inteligência Artificial na Educação Básica, especialmente nos últimos 3 anos. Por outro lado, cerca de $70 \%$ dos artigos científicos publicados na área são artigos exclusivamente de posicionamento (característica $a$ ) os quais levantam as discussões na área e argumentam a favor do ensino e popularização de conhecimentos da IA como extremamente relevantes no contexto de desenvolvimento tecnológico que a sociedade está inserida atualmente e, consequentemente, para o desenvolvimento integral dos estudantes da Educação Básica. A maioria destes trabalhos ressaltam a necessidade de se considerar e ensinar questões éticas e humanas envolvidas com o avanço da tecnologia [Pimentel et al. 2018].

Ademais, as propostas de pesquisa primária com apresentação de resultados de aplicação de recurso educacional e/ou proposta metodológica para o ensino da IA na educação básica ainda são raros. Contudo, os poucos trabalhos que se enquadram nestas características apresentam resultados bastante promissores. Neste sentido, destaca-se o trabalho de número 15 [Rodríguez-Garcia et al. 2019]. Nesse trabalho é apresentada uma revisão bibliográfica do ensino do Aprendizado de Máquina (AM), evidenciando a conexão entre a IA e o PC. Os autores apresentam uma plataforma web (ML4K) desenvolvida por eles para compartilhamento de recursos do ensino do AM na Educação Básica e, especialmente para crianças. Os recursos podem ser exportados para Scratch, App Inventor, ou código Python. Por fim, naquele trabalho são discutidos os resultados da aplicação de recursos educacionais para reconhecimento de voz e desenvolvimento de assistente virtual, em diferentes níveis de escolaridade. Os resultados foram promissores, porém os autores ressaltam que o ensino do Aprendizado de Máquina na educação básica ainda é uma área de estudo muito incipiente.

\section{Considerações finais (iniciais)}

Este artigo apresentou uma discussão inicial e os resultados de uma pesquisa sistemática com o objetivo geral de quantificar e caracterizar os trabalhos científicos na área do ensino da IA na Educação Básica (EB). A discussão inicial apresentou argumentações favoráveis ao ensino 
IX Congresso Brasileiro de Informática na Educação (CBIE 2020)

Anais do XXXI Simpósio Brasileiro de Informática na Educação (SBIE 2020)

e popularização da IA na EB brasileira fundamentadas nas competências e habilidades da BNCC. Já os resultados da revisão sistemática apontam para a potencialidade de pesquisas aplicadas ao ensino da IA na EB, especialmente no Brasil.

\section{Referências}

BBC, B. (2018) "Computational Thinking". Disponível em: $<$ https://www.bbc.com/bitesize/topics/z7tp34j>. Acesso em 21/06/2020.

Brackmann, C. P. 2017, "Desenvolvimento do pensamento computacional através de atividades desplugadas na educação básica". 2017. 224f. Tese (Doutorado em Informática na Educação) Universidade Federal do Rio Grande do Sul, Porto Alegre.

Brasil, MEC. (2018) "BNCC - Base Nacional Comum Curricular". Disponível em: $<\mathrm{http}$ //basenacionalcomum.mec.gov.br>. Acesso em: 02/05/2020.

Brasil, MEC. (2019) “Temas Contemporâneos Transversais na BNCC”. Brasília/DF. Disponível em: $<$ http://basenacionalcomum.mec.gov.br/images/implementacao/contextualizacao_temas_contemp oraneos.pdf $>$. Acesso em 02/05/2020.

Brasil, MEC. (2020a) "Secretaria de Educação Básica”. MEC, Brasília/DF, 2020. Disponível em: $<$ https://www.gov.br/mec/pt-br/acesso-a-informacao/institucional/secretarias/secretaria-deeducacao-basica $>$, acesso em 02/05/2020.

Brasil, CAPES. (2020b) "Portal de Periódicos CAPES/MEC". Disponível em: $<$ https://www.periodicos.capes.gov.br/>, acesso em 01/07/2020.

Canto, K. e Scartezini, T. 2020 "Modelos matemáticos e inteligência artificial são aliados no combate ao coronavírus", Com Ciência, Maio 2020. Disponível em <http:/www.comciencia.br/modelosmatematicos-e-inteligencia-artificial-sao-aliados-no-combate-ao-coronavirus-mas-tambempreocupam-em-relacao-as-questoes-de-privacidade/>, acesso em 15/06/2020.

CIMM. 2020 "Inteligência Artificial chega à grade do Ensino Técnico do Brasil”, Portal Indústria 4.0, 2020. Disponível em: $<$ https://www.industria40.ind.br/noticias/19327-inteligencia-artificial-chegaa-grade-do-ensino-tecnico-do-brasil $>$, acesso em 02/05/2020.

CIEB. (2020) “Portal CIEB” Disponível em: < http://cieb.net.br>, acesso em: 02/05/2020.

Darwiche, A. (2010) "Bayesian networks." Communications of the ACM 53 (12): 80-90. 10. $1145 / 1859204.1859227$.

Freire, P. (1970) "Pedagogy of the Oppressed”. New York: Continuum. 125.

Gomes, A. e Balmant, O. (2019) "Inteligência artificial nos colégios. Portal Terra, 2019. Disponível em: $\quad<$ https://www.terra.com.br/noticias/educacao/inteligencia-artificial-noscolegios,23a7218c38451cd3ccbe2a0e25c490094d9v9aar.html>, acesso em: 02/05/2020.

Gough, D., Oliver, S., e Thomas, J. (2017) “An introduction to systematic reviews”, $2^{\mathrm{a}}$ ed., Los Angeles: SAGE.

INEP. (2019) "Programa Internacional de Avaliação de Estudantes (Pisa)", 2019. Disponível em: http://portal.inep.gov.br/pisa, acesso em 02/05/2020.

ISTE. (2020) "Operational Definition of Computational Thinking for K-12 Education”. Disponível em: $\quad<$ https://id.iste.org/docs/ct-documents/computational-thinking-operational-definitionflyer.pdf>. Acesso em: 02/05/2020.

Jezuz, D. A. F.; Oliveira, J. C. R. e Tortola, E. (2016) "Uma proposta para o ensino do Teorema de Bayes na perspectiva da resolução de problemas". In: Anais do XII Encontro Nacional de Educação Matemática, São Paulo, Julho, 2016. Disponível em: $<$ http://www.sbem.com.br/enem2016/anais/pdf/7478_3221_ID.pdf>, acesso em 15/06/2020. 
IX Congresso Brasileiro de Informática na Educação (CBIE 2020)

Anais do XXXI Simpósio Brasileiro de Informática na Educação (SBIE 2020)

Levy, P. (1993) "Tecnologias da Inteligência: O futuro do pensamento na era da informática" [Technologies of Intelligence: the future of thinking in the informatics era]. Rio de Janeiro, Ed. 34.

Loftus, M. e Madden, M. (2020) "A pedagogy of data and Artificial Intelligence for student subjectification". Teaching in Higher Education. 25. 456-475. 10.1080/13562517.2020.1748593.

Mcelwee, K. (2019) "From math to meaning: Artificial intelligence blends algorithms and applications". Princeton, University, 2019.

Meira, R. (2017) "Pensamento computacional na educação básica: uma proposta metodológica com jogos e atividades lúdicas".2017. 119f. Dissertação (Mestrado Profissional em Tecnologias Educacionais em Rede) - Universidade Federal de Santa Maria, Santa Maria/RS.

Moreira, F.; Mesquita A. e Peres, P. (2019) "Educação 4.0 e a transformação dos ambientes de aprendizagem: O Modelo Personalizado de Ambiente de Aprendizagem 4.0". In: 14th Iberian Conference on Information Systems and Technologies (CISTI), Coimbra, Portugal, 2019, pp. 1-6. Doi: 10.23919/CISTI.2019.8760900.

Olivo, C.; Santin, A. e Oliveira, L. (2015). “Abordagens para Detecção de Spam de E-mail”. LivroTexto dos minicursos do XV Simpósio Brasileiro em Segurança da Informação e de Sistemas Computacionais - SBSeg 2015. Florianópolis/SC, Novembro de 2015. Disponível em: $<$ https://secplab.ppgia.pucpr.br/files/papers/2015-7.pdf>, acesso em: 15/06/2020.

OCDE. (2020) "PISA 2021 Assessment and Analytical Framework. Disponível em: $<$ http://www.oecd.org/pisa/publications/pisa-2021-assessment-and-analytical-framework.htm>, acesso em: 01/07/2020.

Pimentel, C. S.; Queiroz, R. L. ; Lima, P. M. V. ; Sampaio, F. F. (2018) "Projeto Frankie: uma proposta para o ensino de Inteligência Artificial na Educação Básica”. In: XIII Congresso Internacional Informática Educativa (TISE), Nuevas Ideas en Informatica Educativa, Brasília, 2018.

Rodríguez-García, J. D., Moreno-León, J., Román-González, M., e Robles, G. (2019) "Developing Computational Thinking at School with Machine Learning: An exploration". In 2019 International Symposium on Computers in Education (SIIE) (pp. 1-6). IEEE.

Russell, S; Norvig, P. (2020) “Artificial Intelligence: A Modern Approach”. Pearson, 2020. 1136 p.

Soares, C. G.; Souza, D. C.; Castro, J. B. (2018) "Recursos Educacionais Digitais e o ensino de adição e subtração: a concepção de um jogo na perspectiva da Teoria dos Campos Conceituais." VII Congresso Brasileiro de Informática na Educação (CBIE 2018), Anais do VII Congresso Brasileiro de Informática na Educação.

SBC, Sociedade Brasileira da Computação (2019a) "Ensino de Computação na Educação Básica". Disponível em: <http://www.sbc.org.br/documentos-da-sbc/send/203-educacao-basica/1220-bnccem-itinerario-informativo-computacao-2, acesso em: 02/05/2020.

SBC, Sociedade Brasileira da Computação. (2019b) "Itinerário formativo da Computação". Disponível em: $\quad<$ http://www.sbc.org.br/documentos-da-sbc/send/203-educacao-basica/1216-itinerarioformativo-da-computacao>, acesso em: 02/05/2020.

CEIE, Comissão Especial de Informática na Educação da SBC (2020) "Portal de Publicações da CEIE". Disponível em $<$ https://www.br-ie.org/pub/>, acesso em 01/07/2020.

Valente, J. A. (2016) "Integração do Pensamento Computacional no Currículo da Educação Básica: Diferentes Estratégias Usadas e Questões de Formação de Professores e Avaliação do Aluno". Revista eCurriculum, v.14, n.3, p.5562, 2016.

Velazco, C. (2018) "Data from wearables helped teach an AI to spot signs of diabetes". Engadget, 2018. Disponível em: <https://www.engadget.com/2018-02-07-deepheart-diabetes-cardiogram-ai.html>, acesso em 15/06/2020.

Wing, J. M. (2006) “Computational Thinking”. Communications of the ACM, v.49, 3, p. 33-35, 2006. 